\title{
Epiphyseal Arterial Network and Inferior Retinacular Artery Seem Critical to Femoral Head Perfusion in Adults With Femoral Neck Fractures
}

\author{
Dewei Zhao MD, PhD, Xing Qiu PhD, Benjie Wang MD, \\ Zihua Wang MD, Wei Wang PhD, Jun Ouyang PhD, Rona M. Silva PhD, \\ Xiaotian Shi PhD, Kai Kang PhD, Dachuan Xu PhD, Chuang Li MD, \\ Shizhen Zhong PhD, Yu Zhang PhD, Kent E. Pinkerton PhD
}

Received: 17 October 2016/Accepted: 9 March 2017/Published online: 17 March 2017

(C) The Association of Bone and Joint Surgeons (B) 2017

\begin{abstract}
Background A better understanding of the blood supply of the femoral head is essential to guide therapeutic strategies for patients with femoral neck fractures. However, because of the limitations of conventional techniques,
\end{abstract}

Funding was provided by the Chinese National Natural Science Foundation (DZ) (81371942), National Science and Technology Support Program (DZ) (2012BAI17B02), and the NHFPC special fund for health scientific research for the public welfare (DZ) (201402016).

All ICMJE Conflict of Interest Forms for authors and Clinical Orthopaedics and Related Research ${ }^{\mathbb{R}}$ editors and board members are on file with the publication and can be viewed on request.

Clinical Orthopaedics and Related Research ${ }^{\mathbb{R}}$ neither advocates nor endorses the use of any treatment, drug, or device. Readers are encouraged to always seek additional information, including FDAapproval status, of any drug or device prior to clinical use. Each author certifies that his or her institution approved the human protocol for this investigation, that all investigations were conducted in conformity with ethical principles of research, and that informed consent for participation in the study was obtained.

This work was performed at the Affiliated Zhongshan Hospital of Dalian University, Dalian, Liaoning, China, and the Southern Medical University, Guangzhou, Guangdong, China.

Electronic supplementary material The online version of this article (doi:10.1007/s11999-017-5318-5) contains supplementary material, which is available to authorized users.

D. Zhao ( $₫)$, X. Qiu, B. Wang, Z. Wang, W. Wang, K. Kang, C. $\mathrm{Li}$

Department of Orthopedics, Affiliated Zhongshan Hospital of Dalian University, No.6 Jiefang Street, Dalian 116001, Liaoning, China

e-mail: gksys3509@foxmail.com; zhaodewei2016@163.com

D. Zhao, X. Qiu, J. Ouyang, D. Xu, S. Zhong

Department of Anatomy, Guangdong Provincial Key Laboratory of Medical Biomechanics, School of Basic Medicine Science, Southern Medical University, Guangzhou, Guangdong, China the precise distribution and characteristics of intraosseous arteries of the femoral head are not well displayed.

Questions/purposes To explore the characteristics and interconnections of the intraosseous vessel system between different areas of the femoral head and the possible blood supply compensatory mechanism after femoral neck fracture.

Methods The three-dimensional (3-D) structures of the intraosseous blood supply in 30 uninjured normal human femoral heads were reconstructed using angiography methods and microCT scans. The data were imported in the AMIRA ${ }^{\circledR}$ and MIMICS ${ }^{\circledR}$ software programs to reconstruct and quantify the extra- and intraosseous arteries (diameter, length). In a separate experiment, we evaluated the residual blood supply of femoral heads in 27 patients with femoral neck fractures before surgery by analyzing digital subtraction angiography data; during the study period, this was performed on all patients in whom hip-preserving surgery was planned, rather than arthroplasty. The number of affected and unaffected subjects included in the three groups (superior, inferior, and anterior retinacular arteries) with different types of fractures (Garden Types I-IV) were recorded and analyzed (Fisher's exact test) to reflect the affected degrees of these three groups of retinacular arteries in patients after femoral neck fractures.

X. Qiu, R. M. Silva, K. E. Pinkerton

Center for Health and the Environment, University of California, Davis, CA, USA

X. Shi

Department of Anatomy, Hainan Medical College, Haikou,

Hainan, China

Y. Zhang

General Hospital of Guangzhou Military Region, Guangzhou,

Guangdong, China 
Results The main results of our cadaver study were: (1) the main blood supply sources of the femoral head were connected by three main network structures as a whole, and the epiphyseal arterial network is the most widely distributed and the primary network structure in the femoral head; (2) the main stems of the epiphyseal arteries which were located on the periphery of the intraosseous vascular system have fewer anastomoses than the network located in the central region; (3) compared with the round ligament artery and anterior retinacular artery, the inferior retinacular artery has a relatively large caliber. Digital subtraction angiography of the 27 patients with hip fractures indicated that the inferior retinacular arterial system had a high likelihood of being unaffected after femoral neck fracture (100\% [14 of 14] in nondisplaced fractures and 60\% [six of $10]$ in Garden Type III fractures).

Conclusions The epiphyseal arterial network and inferior retinacular arterial system appear to be two important structures for maintaining the femoral head blood supply after femoral neck fracture. Increased efforts to protect these key structures during surgery, such as drilling and placing internal implants closer to the central region of the femoral head, might be helpful to reduce the effect of iatrogenic injury of the intraosseous vascular system.

Clinical Relevance 3-D anatomic evidence of intraosseous arterial distribution of the femoral head and the high frequency with which the inferior retinacular arteries remained patent after femoral neck fracture lead us to consider the necessity of drilling and placing internal implants closer to the central region of the femoral head during surgery. Future controlled studies might evaluate this proposition.

\section{Introduction}

Femoral neck fractures are common; approximately 1.7 million patients in the United States sustain these injuries each year [15]. The most severe complication of femoral neck fracture is femoral head necrosis (osteonecrosis of the femoral head) [6]. In adult patients, the reported incidence of femoral neck fracture-related osteonecrosis ranges from $12 \%$ to $86 \%$ owing to the various severities of the femoral neck fractures and/or the surgical procedure $[1,4,13]$. The currently accepted etiologic theory of traumatic avascular necrosis is that a displaced femoral neck fracture may lead to rupture, distortion, and compression of the blood vessels supplying blood to the femoral head, thus causing avascular necrosis [6, 23]; however, some patients with a displaced femoral neck fracture do not have osteonecrosis develop, possibly owing to the existence of the compensatory blood supply architecture of the femoral head [20]. By contrast, some patients with nondisplaced fractures also may have osteonecrosis develop [5, 16], which appears to indicate that many factors other than fracture displacement may influence the blood supply.

A better understanding of the blood supply of the femoral head therefore is essential to guide the therapeutic strategy for patients with a femoral neck fracture. However, compared with the extraosseous blood supply, previous evidence regarding the intraosseous blood supply system of the femoral head is relatively insufficient [2, 4, 20, 22, 23], and requires further exploration in depth. By using the conventional technology of perfusion imaging (Spalteholz method) [19], general information regarding the intraosseous vascular structure can be acquired; such studies have verified that the blood vessels in femoral heads consist primarily of round ligament arteries, lateral epiphyseal arteries, and inferomedial metaphyseal arteries, and have provided fragmentary evidence of anastomosis among different regions of the intraosseous vascular system [4, 20-23]. Boraiah et al. [2] confirmed the existence of this anastomosis and further emphasized the importance of the inferior retinacular artery for femoral head blood perfusion by using gadolinium-enhanced MRI. Nevertheless, because of the abundance of capillaries and overlapping areas, the specific three-dimensional (3-D) morphologic characteristics of these anastomoses and the spatial relationship among the various parts are difficult to precisely ascertain, whether using the Spalteholz technique or MRI.

In the current study, we used a new technique using microCT and 3-D reconstruction technology to verify the blood supply structure of the femoral head in a cadaver study of uninjured human hips to show the 3-D intraosseous vascular structures, study the characteristics of the extra- and intraosseous vascular anastomoses among different blood supply sources, and measure the extra- and intraosseous vessels of the femoral head. In addition, the blood supply after a femoral neck fracture was assessed by digital subtraction angiography (DSA) imaging in 27 patients with displaced and nondisplaced femoral neck fractures to verify the affected situation of the retinacular arterial system, thus to delineate the possible compensatory blood supply pathway of the femoral head after femoral neck fracture.

\section{Materials and Methods}

\section{Normal Human Femoral Head Arterial Anatomy}

\section{Fresh Cadaver Specimens}

Three-dimensional imaging of the vascular supply in the femoral head was obtained and reconstructed in 30 hips of patients without a history of hip disease or surgery (Chinese ethnicity; 24 males; age, 25-67 years). Approval from 
the ethics committee of Southern Medical University was obtained.

\section{Barium Sulfate Perfusion}

A contrast agent $\left(30 \% \mathrm{BaSO}_{4}\right.$ in a $5 \%$ gelatin suspension, Catalog No. V900863; Sigma, St Louis, MO, USA) was injected in the femoral artery to observe the arterial distribution of the femoral head at a high resolution [9, 20, 21] A constant machine-controlled perfusion pressure (130$140 \mathrm{~mm} \mathrm{Hg}$ ) was maintained for at least 20 minutes; the mean size of the $\mathrm{BaSO}_{4}$ particles was $0.7 \mu \mathrm{m}$. To prevent the contrast agent from spreading to lower limb tissues, a tourniquet was placed at the mid-thigh. After perfusion, the femoral head was removed.

Microangiography, 3-D Reconstruction, Observation, and Measurement

MicroCT (Inveon $^{\text {TM }}$ Multi Modality; Siemens Medical Solutions, Inc, Malvern, PA, USA) was used to observe the arterial architecture of the femoral heads. For each specimen, $1024 \mathrm{CT}$ images were acquired along the longitudinal axis with a resolution of $23.47 \mu \mathrm{m}$. All individual CT images of the femoral head were reconstructed to observe the intraosseous arterial distribution in three dimensions using image analysis software (Inveon ${ }^{\mathrm{TM}}$ Image Research Workplace; Siemens Medical Solutions, Inc). The course, branch, distribution, and anastomosis of the extra- and intraosseous vessels were observed on 30 reconstructed 3$\mathrm{D}$ digitized vascular models. The quantitative data of the femoral head vascular system (the arterial caliber, arterial length) were measured and quantified by importing the digitized 3-D data into AMIRA ${ }^{\circledR}$ 5.4.3(FEI Visualization Sciences Group, Hillsboro, OR, USA) and MIMICS ${ }^{\circledR} 14.0$ (Materialise Europe, Leuven, Belgium).

The Landmarks for Locating the Extra- and Intraosseous Vessels

The femoral head was divided into different regions using four bony landmarks to better understand the distribution of the femoral neck arteries (Fig. 1). The four landmarks included the greater tubercle and the lesser trochanter (the upper and lower ends of the intertrochanteric line, respectively), the quadrate tubercle, and the lesser tubercle. The anatomic lines of the greater tubercle, the lesser trochanter, the quadrate tubercle, and the lesser tubercle also were used.
In addition, the epiphyseal scar was used as a landmark to locate the intraosseous vascular structures on the 3-D reconstructed femoral head images.

Observation and Evaluation of the Femoral Head Blood Supply after Femoral Neck Fracture in Adults Based on DSA Imaging

A total of 27 adult patients (age range, 19-76 years) hospitalized at our facility between October 2013 and August 2016 with a diagnosis of a femoral neck fracture and who underwent DSA were included in the study. All patients provided written, informed consent for the study and a separate consent for the surgical procedure. This part of the experiment was approved by the ethics committee of Affiliated Zhongshan Hospital of Dalian University, and was registered in the Chinese Clinical Trial Registration system (Number: ChiCTR-IPR-15006607).

DSA is not the routine examination for every patient with a femoral neck fracture in our hospital. However, for patients whom we think would benefit from a joint-preserving treatment rather than THA (mainly considering the age, physical condition, and patient's wishes, regardless of the classification of the fracture), DSA is performed to evaluate the blood supply situation of the affected femoral neck and head, to develop an appropriate joint-preserving therapeutic strategy.

\section{Inclusion Criteria for DSA Examination}

For patients 60 years or younger, we routinely required DSA examinations. In addition, for some patients older than 60 years who engaged in more physical activity and were in good physical condition, we also required DSA examinations. These patients were considered to benefit more from a native joint-preserving treatment rather than a THA.

The patients should be in relatively good physical condition without any severe complex disease or injury, should consent to invasive examination, and should provide informed consent and study consent.

All included patients underwent traction of the affected limb after a definitive diagnosis by radiographs and CT (patients with Garden Types III and IV fractures underwent closed reduction before limb traction). The DSA examination was completed after a preliminary closed reduction and limb traction within 12 to 24 hours after hospitalization. The residual blood supply of the femoral head in these patients was evaluated based on the DSA images. In addition, the fracture classifications (Garden Types I-IV) were determined by preoperative radiographs and CT images. 


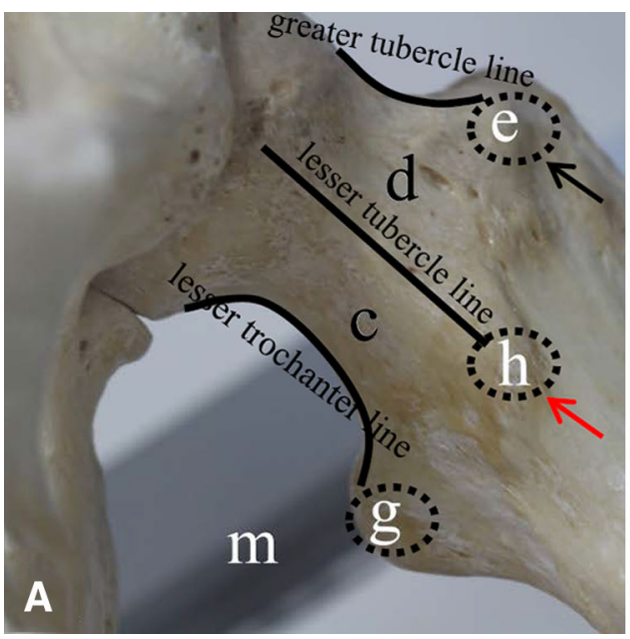

Fig. 1A-B Four bony landmarks are used to divide the femoral neck for better understanding of the distribution of the femoral neck arteries. (A) The femoral greater tubercle (e, black arrow), lesser tubercle ( $h$, red arrow), and lesser trochanter ( $g$ ) from an anteromedial view of the proximal femur, along with their corresponding lines(the greater tubercle line, lesser tubercle line, and lesser trochanter line) that parallel to the long axis of the femoral neck are shown. $\mathrm{d}=$ the region between the femoral greater and lesser tubercle lines; $\mathrm{c}=$ the region between the lesser tubercle and lesser trochanter lines. (B) The quadrate tubercle (f, blue arrow) and lesser trochanter (g) are shown

There were 146 patients with femoral neck fractures hospitalized and treated at our facility between October 2013 and August 2016 (64 patients younger than 60 years), according to the inclusion criteria; 28 patients finally underwent DSA examinations (including three patients older than 60 years who were eligible for a native jointpreserving treatment). Among these 28 patients with DSA examinations, 27 were included in this study (one patient who was 15 years old was excluded from the final statistics).

Observation Methods and Evaluation Standards of the DSA Imaging

With DSA imaging, an "unaffected" vessel should be developed intact, without interruption, blurring, and eventually enter the femoral head, otherwise, it would be regarded as "affected".

All imaging data (DSA and CT images, and radiographs) were evaluated separately by two radiologists (CL, $\mathrm{KK}$ ) and three senior orthopaedic surgeons (DZ, BW, and $\mathrm{ZW}$ ), the results were combined for comparison, the inconsistent results were discussed, and a consensus ultimately was reached.

We compared the proportion of residual blood supply in two different fracture types (nondisplaced and displaced), and the unaffected proportion among the three groups of

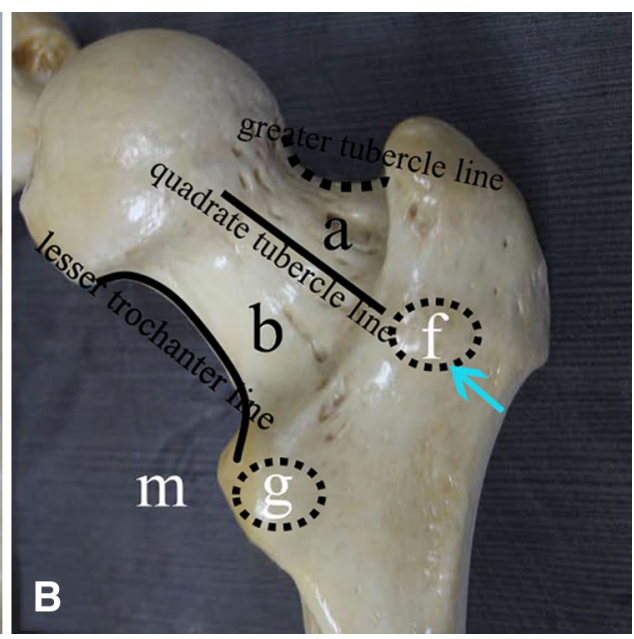

from a posterior view of the proximal femur. The corresponding lines of the quadrate tubercle and lesser trochanter, which parallel the long axis of the femoral neck are marked in black lines, respectively, while the perspective greater tubercle line is marked with a black dashed line. The cartilaginous edge of the femoral head appears as two prominent angles that intersect with the quadrate and greater tubercle lines of the femur. $b=$ the region between the lesser trochanter and quadrate tubercle lines; $\mathrm{a}=$ the region between the quadrate and greater tubercle lines; $\mathrm{M}=$ medial anatomic direction.

retinacular arteries. A probability less than 0.05 using Fisher's exact test was considered statistically significant.

\section{Results}

Normal Human Femoral Head Arterial Anatomy

\section{Intraosseous Arterial Network of the Femoral Head}

The femoral head receives its primary blood supply from the superior, anterior, and inferior retinacular arteries and the round ligament arteries (Fig. 2). The branches of three retinacular arterial groups (superior, inferior, and anterior groups) constitute the main stems of the epiphyseal and metaphyseal arterial branches after entering the femoral head and then anastomose with each other from the periphery to the center to form an epiphyseal arterial network above the epiphyseal scar (Video 1. Supplemental materials are available with the online version of $C O R R^{\circledR}$.) and a metaphyseal arterial network below the epiphyseal scar. Arteriographic images confirm the presence of these vascular networks. The arterial plexus of the round ligament arterial group entered the femoral head through the fovea and then joined the epiphyseal arterial network structure (Fig. 3), which is the most widely distributed and the primary network structure in the femoral head. 


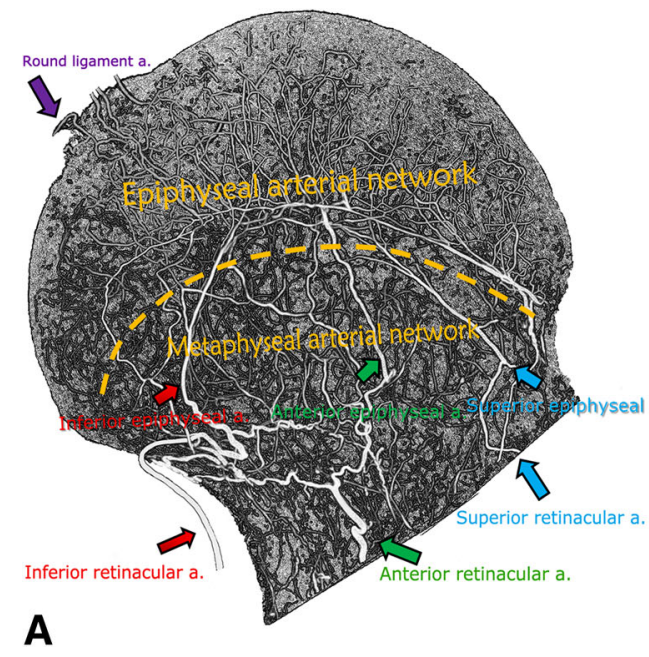

Fig. 2A-B The illustrations show the three groups of retinacular arterial systems (superior, inferior, and anterior) and the round ligament arterial systems which formed the epiphyseal and metaphyseal arterial network after entering the femoral head. (A) A graphic microCT image of the femoral head after arteriographic injection and (B) a schematic diagram are shown. The superior retinacular arteries gives off superior epiphyseal (small light blue arrow, A; solid light blue line, B) and superior metaphyseal (broken light blue line, B) arteries that begin at the epiphyseal scar level (broken yellow line, A; broken black line, B). The inferior retinacular arteries gives off inferior epiphyseal (red arrow, A; solid red line, B) and inferior metaphyseal (broken red line, B) arteries that begin below the

\section{Retinacular, Epiphyseal, and Metaphyseal Arteries}

The branches of the superior, anterior, and inferior retinacular arteries enter the femoral head and then branch off the main stem of the epiphyseal and metaphyseal branches, which extend to combine the epiphyseal arterial network and metaphyseal arterial network, respectively.

The superior retinacular arteries enter the femoral neck between the greater and quadrate tubercle lines (Fig. 1A, area A), typically as three to six arterial branches that initially run linearly. After entry in the femoral head at the epiphyseal scar level (Fig. 3A), these arteries become the main stem of the superior epiphyseal arteries that anastomose with the inferior and anterior epiphyseal arterial branches (Fig. 4) to form the epiphyseal arterial network. Their arterial path closely and superficially follows along the surface of the old epiphyseal scar (Video 1. Supplemental material is available with the online version of $C O R R^{\circledR}$.). The distribution of branches from the epiphyseal arterial network is directed toward the articular surface of the femur and separated by approximately $15 \mathrm{~mm}$ such that their initial orientation is perpendicular to the epiphyseal scar and the articular surface of the femoral head. These branches are consecutively and radially arranged into one to three arterial arches (Fig. 5), with adjacent arteries running approximately parallel to each other. A

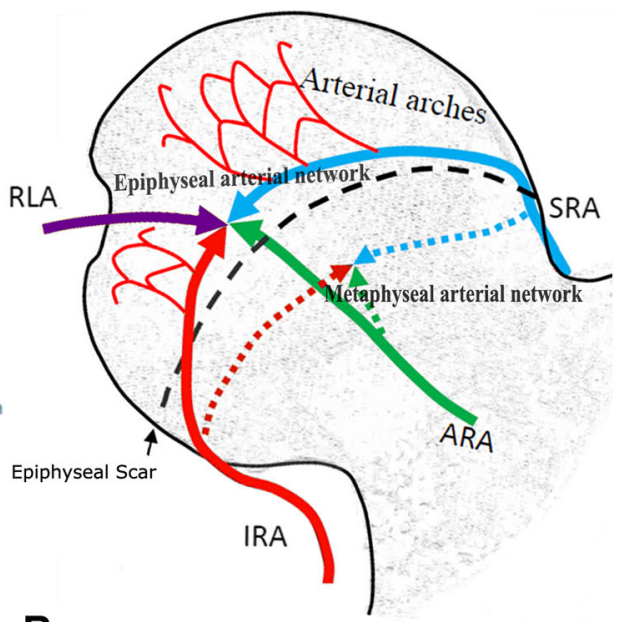

B

epiphyseal scar. The femoral epiphyseal arterial network is formed by epiphyseal branches of the superior and inferior retinacular arteries, and anterior retinacular arteries (green arrow, A; solid green line, B) above the epiphyseal scar along with the branches of the round ligament arteries (purple arrow, A; solid purple line, B). The metaphyseal arterial network is formed by metaphyseal branches of the superior, inferior, and anterior arteries (broken light blue line, red and green lines, B) in the metaphysis (below the epiphyseal scar). RLA = round ligament artery; SRA = superior retinacular artery; IRA = inferior retinacular artery; ARA = anterior retinacular artery.

communicating branch, typically of the same diameter, connects both sides of the arterial arches (Fig. 5). Although one to three tiers of arterial arches can be found in the epiphysis lying between the epiphyseal arterial network and the articular cartilage, the most common arrangement is two tiers. This arterial plexus arises in a diverging pattern from the arching intraosseous arteries in the epiphysis. Generally, the main stems of epiphyseal arteries are located on the periphery of the intraosseous vascular system. The secondary branches and arterial arches typically run from the periphery to the center and form the epiphyseal arterial network, resulting in a "dome shape" above the epiphyseal scar. Structures closer to the center contain a greater number of anastomoses (Fig. 4). However, in the superolateral region, the superior epiphyseal arteries anastomose in the epiphysis with fewer secondary anastomoses and arches than in other regions of the femoral head (Fig. 5). This region represents the most-critical site where osteonecrosis and resultant collapse occur [3]. By contrast, metaphyseal arterial branches frequently arise from branches of the superior, anterior, and inferior retinacular arteries in the femoral head, anastomose in the metaphysis to form the metaphyseal arterial network (Figs. 2 and 3), and anastomose secondarily with the intraosseous arteries of the femoral neck. Unlike arterial structures in the epiphysis, metaphyseal anastomoses are fewer in quantity and 
Fig. 3A-D The illustrations show the microCT images of the femoral head after arteriographic injection. (A) Coronal and (B) sagittal views show the position relationship among retinacular arteries, epiphyseal arteries, and the epiphyseal scar. The epiphyseal scar (black line) separates the epiphyseal and metaphyseal circulations. The epiphyseal arterial network (spanned by the dotted yellow line) lies immediately above the scar, whereas the metaphyseal network lies below. $\mathrm{m}, 1, \mathrm{a}$, and $\mathrm{p}=$ medial, lateral, anterior, and posterior anatomic directions, respectively. $\uparrow, \boldsymbol{\Delta}, \Delta=$ superior, inferior, and anterior metaphyseal arteries, respectively. (C) Coronal and (D) sagittal views show that the epiphyseal arterial stems of round ligament arterial system joined the epiphyseal arterial network in the femoral head. RLA = round ligament artery; SRA = superior retinacular artery; IRA = inferior retinacular artery; ARA $=$ anterior retinacular artery; SEA = superior epiphyseal artery; IEA $=$ inferior epiphyseal artery.

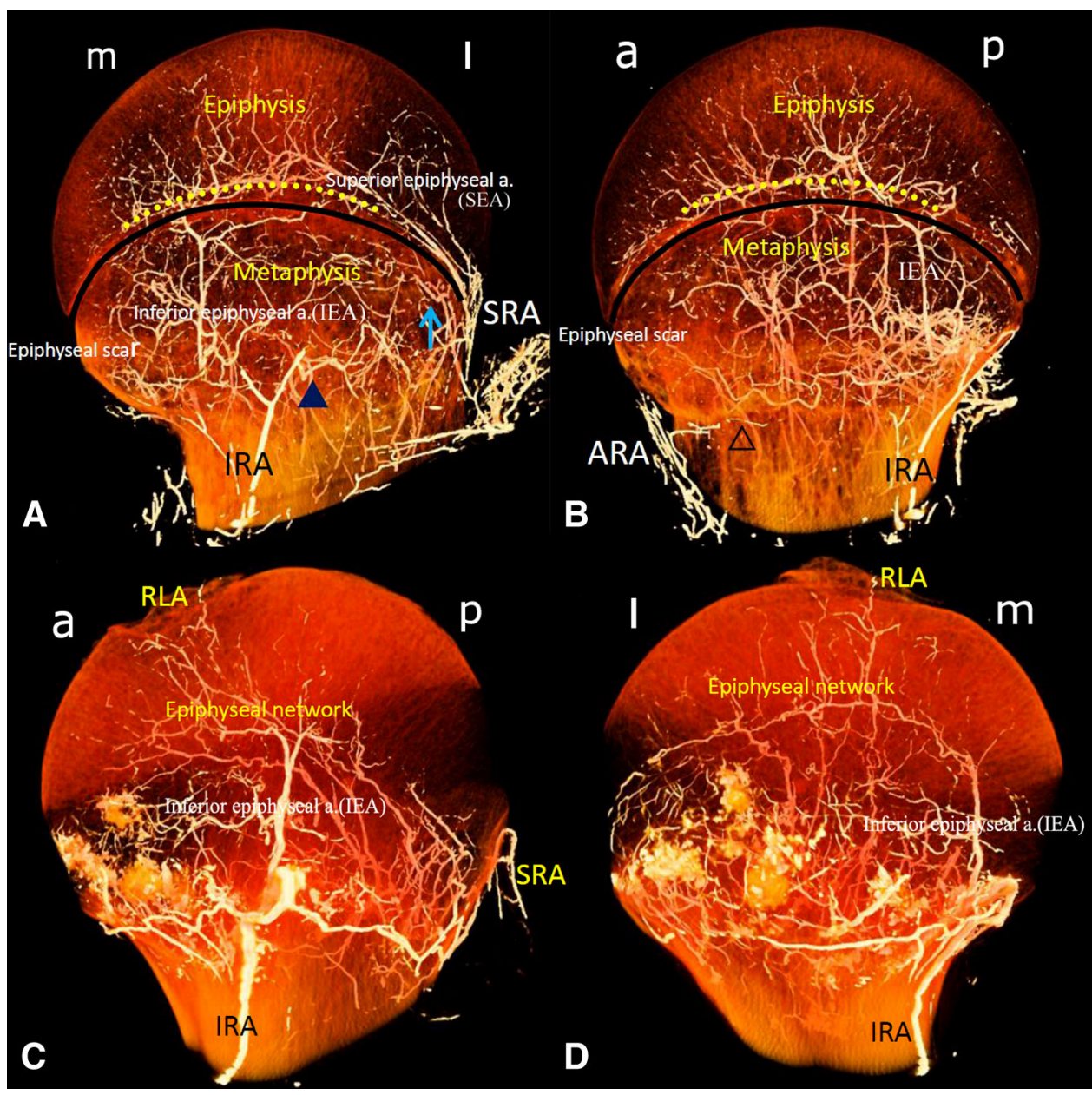

lack arterial arches. Similar to the superior arteries, the inferior arteries are prominent and easily observed (Figs. 2 and 3). One to three inferior retinacular arteries are present, with a mean diameter of $0.62 \mathrm{~mm}$ (similar to the superior retinacular arteries; mean value, $0.66 \mathrm{~mm}$ ) (Table 1), and frequently one inferior retinacular artery vessel exists that is larger than any others. The vessels first pass through the femoral neck between the lesser trochanter line and quadrate tubercle line (Fig. 1A, area B). The vessels subsequently penetrate the femoral head at the posteromedial surface of the femoral neck to enter the bone close to the inferior margin of the articular cartilage, where an area with a $1.6-\mathrm{mm}$ gap (Table 1) is present under the medial cortical bone. By contrast, the superior and the anterior retinacular arteries do not have this gap and are closer to the bone surface. The inferior retinacular artery branches to form the inferior epiphyseal arteries and metaphyseal arteries in the femoral neck under the epiphyseal scar (Fig. 2). The stem of the inferior retinacular arterial then branches, extends up, and penetrates the epiphyseal scar level to join the epiphyseal arterial network (Figs. 2 and 3), whereas the metaphyseal arterial branches join the metaphyseal arterial network under the epiphyseal scar.

The anterior retinacular artery, which passes through the femoral neck between the lesser tubercle and the lesser trochanter lines (Fig. 1B, area C), has a mean diameter of $0.47 \mathrm{~mm}$. The results showed that these vessels occasionally were absent (Table 1). After entering the femoral head at the epiphyseal scar level, the anterior retinacular arteries branch off the metaphyseal and epiphyseal arteries to join the metaphyseal arterial network and epiphyseal arterial network, respectively.

\section{Round Ligament Arteries}

Two to three round ligament arteries are present, with a mean diameter of $0.3 \mathrm{~mm}$ (Table 1). The round ligament arteries always pass into the femoral head. On entry to the fovea, they ramify locally and join with the superior, inferior, and anterior arterial plexuses in the epiphysis (Figs. 2 and 3). This anastomosis was shown by the outflow of $\mathrm{BaSO}_{4}$ medium through round ligament arteries 
Fig. 4A-C (A) Coronal, (B) mediosagittal, and (C) transverse views of the femoral head show the "dome shape" epiphyseal arterial network that formed with the main stems of the three groups of epiphyseal arteries (superior, inferior, and anterior epiphyseal arteries). $\mathrm{m}$, 1, a, and $\mathrm{p}=$ medial, lateral, anterior, and posterior anatomic directions, respectively. The main stems of the epiphyseal arteries are located on the periphery of the intraosseous vascular system; structures closer to the center contain a greater number of anastomoses. The white arrow shows the lateral region of the femoral area, which contains fewer anastomotic connections and arterial arches in contrast to the medial portion of the femoral head. This lateral region is further demarcated with a solid yellow line for clarity.
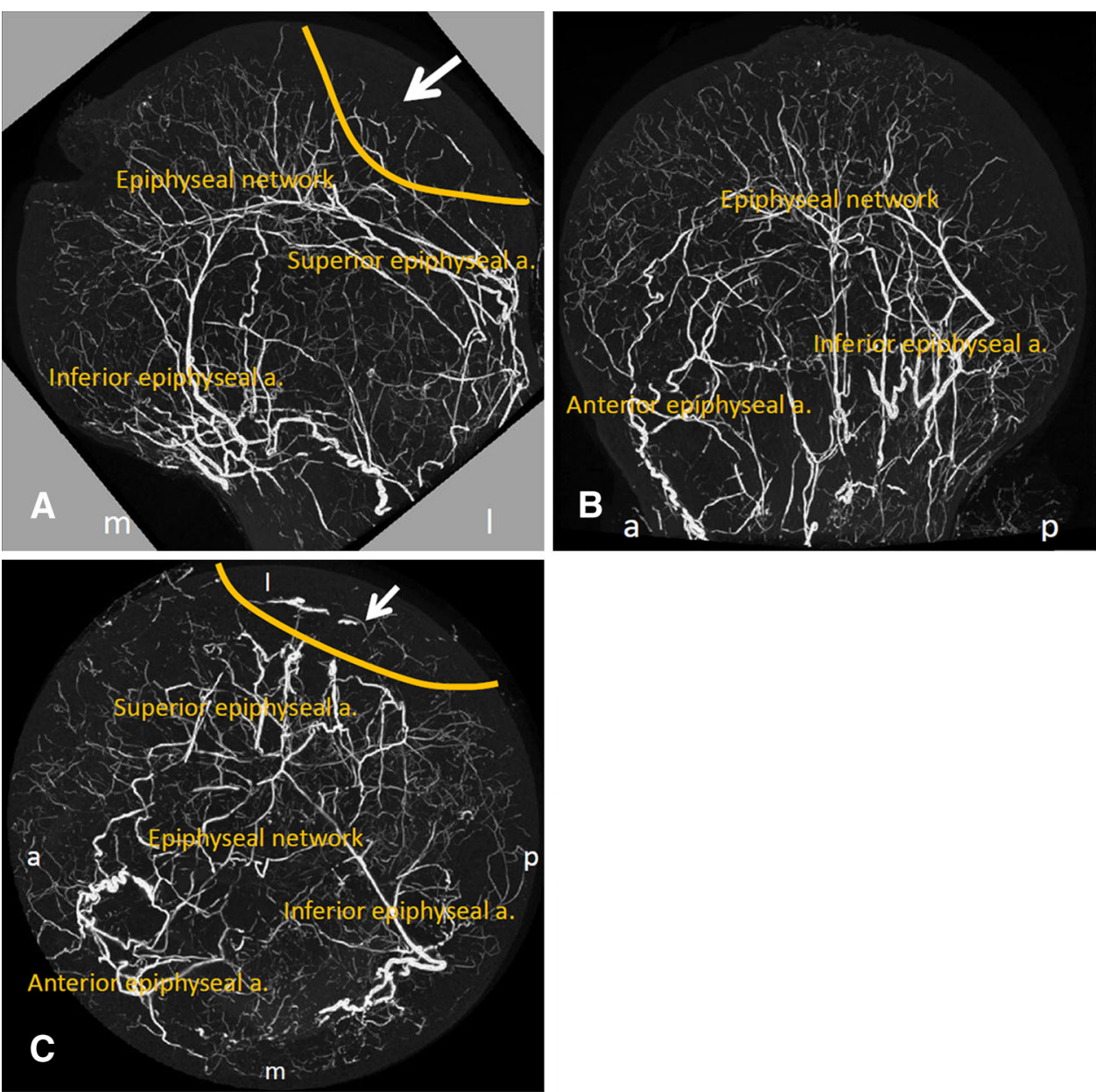

after injection in the femoral artery. In this study, the round ligament arteries exhibited the highest rate of absence and a relatively smaller caliber (Table 1).

\section{Femoral Neck Arterial Networks}

Before the superior, anterior, and inferior retinacular arteries enter the bone, they have frequent interconnecting anastomoses, called the circulus articuli vasculosus, in the subsynovial tissues [8]. The circulus articuli vasculosus is evident at the neck of the femur in all major regions (Fig. 1), except between the lesser trochanter and lesser tubercle lines, where it tends to be deficient (Fig. 6). The superior and inferior retinacular arteries, and the deep branch of the medial femoral circumflex arteries form a Cshaped ring (Fig. 7) around the base of the femoral neck. The anterior retinacular arteries occasionally join this $\mathrm{C}$ shaped ring through the circulus articuli vasculosus (Fig. 6) at the base of the femoral head. The neck of the femur is almost entirely dependent on blood supplied by the circulus articuli vasculosus and this C-shaped ring of arteries (Fig. 8), which enter the superior aspect of the femoral neck mainly between the lines of the lesser trochanter and greater tubercle lines (Fig. 1A, areas A and B) some distance from the edge of the articular cartilage. These arteries penetrate the cervical cortex, follow a straight course downward and sideways to ramify with nutrient arteries of the femur (Fig. 9), which becomes the femoral neck, and then spread out to anastomose with metaphyseal artery branches.

Observation and Assessment of the Blood Supply after Femoral Neck Fracture in 27 Adult Patients Based on DSA Imaging

The preoperative DSA imaging data (Appendix 1. Supplemental material is available with the online version of CORR ${ }^{\mathbb{R}}$.) revealed a high probability that one or more retinacular blood vessels (superior, inferior, or anterior retinacular arteries) were intact (unaffected) in nondisplaced fractures (Garden Types I and II) (Table 2). Overall, the unaffected proportion of the retinacular artery (superior, inferior, and anterior) in nondisplaced fractures was higher than that in displaced fractures $(76 \%$ vs $23 \%$; $p<$ 


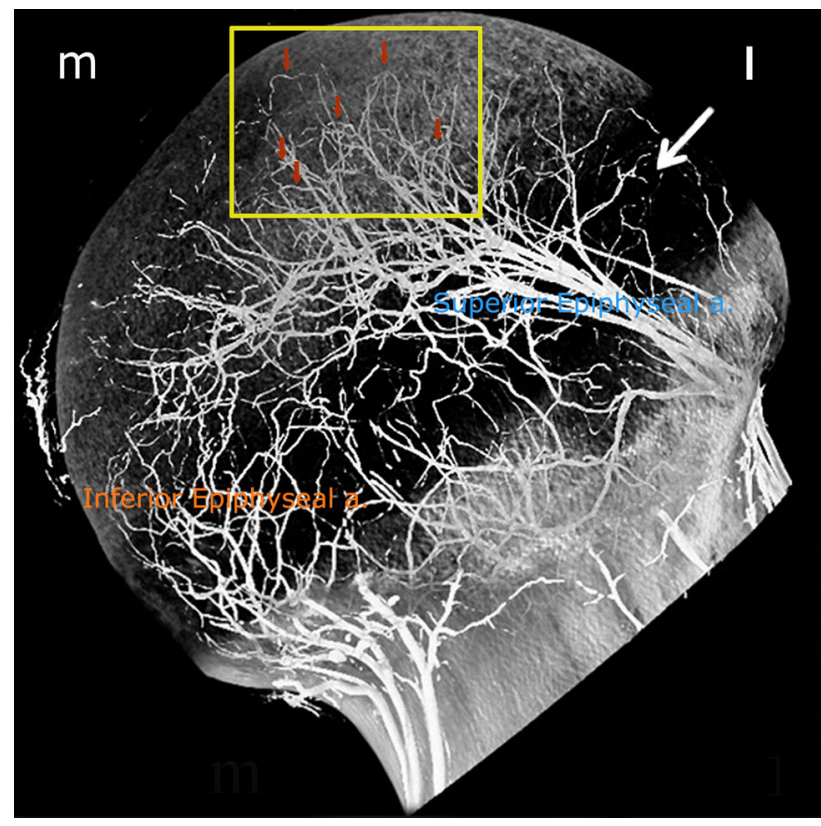

Fig. 5 The arterial arches (yellow box) branching radially from the femoral epiphyseal arterial network are shown. One to three stacks of the same arterial arches (red arrows) originate from the epiphyseal arterial network. The white arrow indicates the lateral (1) region of the femoral head with the fewest anastomotic connections and arterial arches in contrast to the medial $(\mathrm{m})$ region.
0.0001). Moreover, for two of the three retinacular arteries, a higher unaffected proportion was found in nondisplaced fractures (nine of 14 vs 0 of $13, p=0.0006$; inferior retinacular artery, 14 of 14 vs six of $13, p=0.002$; anterior retinacular artery, nine of 14 vs three of $13, \mathrm{p}=0.054$ ).

Remarkably, compared with the superior and anterior retinacular arteries, the inferior retinacular arteries have a prominent unaffected proportion in Garden Types I to III femoral neck fractures (superior retinacular artery [nine of $24]$ vs the anterior retinacular artery [ 12 of 24], $p=0.56$, vs the inferior retinacular artery [20 of 24], $\mathrm{p}=0.003$ ). The unaffected proportion of the inferior retinacular arteries was $100 \%$ (14 of 14) in nondisplaced fractures (Garden Types I-II) and 60\% (six of 10) in Garden Type III fractures.

In Garden Type IV fractures, none of the hips had a residual retinacular artery.

\section{Discussion}

The intra- and extraosseous vascular system of the femoral head and neck in adults has been widely investigated [2, 4, 7, 9-12, 20-24]. However, compared with the extraosseous vascular system, fewer studies exist of the

Table 1. Characterization of diameters or lengths of femoral head arteries*

\begin{tabular}{|c|c|c|c|c|c|c|c|c|c|c|}
\hline Femoral head artery & Number & Minimum & Maximum & Mean & SD & Variance & Median & Quartiles & $\begin{array}{l}\text { Shapiro-Wilk } \\
\text { statistic }\end{array}$ & $\begin{array}{l}\mathrm{p} \\
\text { Value }\end{array}$ \\
\hline \multicolumn{11}{|l|}{ Diameter (mm) } \\
\hline Anterior retinacular artery & 11 & 0.36 & 0.74 & 0.47 & 0.13 & 0.018 & 0.42 & $0.380-0.570$ & 0.865 & 0.167 \\
\hline Anterior epiphyseal artery & 7 & 0.27 & 0.49 & 0.35 & 0.07 & 0.005 & 0.34 & $0.300-0.380$ & 0.952 & 0.753 \\
\hline Anterior metaphyseal artery & 5 & 0.28 & 0.38 & 0.34 & 0.05 & 0.002 & 0.36 & $0.285-0.375$ & 0.846 & 0.181 \\
\hline Inferior retinacular artery & 29 & 0.25 & 1.50 & 0.62 & 0.29 & 0.082 & 0.57 & $0.452-0.693$ & 0.886 & 0.339 \\
\hline Inferior epiphyseal artery & 27 & 0.17 & 1.19 & 0.48 & 0.19 & 0.038 & 0.45 & $0.350-0.550$ & 0.793 & $0.072^{\dagger}$ \\
\hline Inferior metaphyseal artery & 14 & 0.21 & 0.74 & 0.40 & 0.15 & 0.022 & 0.36 & $0.318-0.425$ & 0.905 & 0.212 \\
\hline Superior retinacular artery & 24 & 0.39 & 1.27 & 0.66 & 0.22 & 0.049 & 0.59 & $0.489-0.766$ & 0.907 & 0.225 \\
\hline Superior epiphyseal artery & 28 & 0.25 & 1.00 & 0.48 & 0.16 & 0.025 & 0.43 & $0.357-0.589$ & 0.847 & $0.039^{\dagger}$ \\
\hline Superior metaphyseal artery & 17 & 0.17 & 0.66 & 0.36 & 0.12 & 0.015 & 0.36 & $0.269-0.430$ & 0.998 & 0.908 \\
\hline Round ligament artery & 6 & 0.24 & 0.40 & 0.30 & 0.06 & 0.004 & 0.28 & $0.248-0.348$ & 0.832 & 0.194 \\
\hline Epiphyseal artery networks & 21 & 0.23 & 0.56 & 0.31 & 0.07 & 0.005 & 0.30 & $0.270-0.345$ & 0.977 & 0.948 \\
\hline Circulus articuli vasculosus & 22 & 0.22 & 0.46 & 0.32 & 0.07 & 0.004 & 0.31 & $0.258-0.370$ & 0.946 & 0.464 \\
\hline \multicolumn{11}{|l|}{ Length $(\mathrm{mm})$} \\
\hline $\begin{array}{l}\text { Epiphyseal arterial network to } \\
\text { femoral head artery }\end{array}$ & 23 & 10.45 & 18.89 & 14.67 & 2.07 & 4.268 & 14.53 & $13.350-15.480$ & 0.965 & 0.858 \\
\hline Superior epiphyseal artery & 22 & 12.23 & 19.32 & 14.84 & 1.32 & 1.754 & 14.70 & $14.375-14.995$ & 0.848 & 0.117 \\
\hline Inferior gap & 28 & 0.24 & 3.25 & 1.64 & 0.75 & 0.559 & 1.65 & $1.045-2.198$ & 0.892 & 0.288 \\
\hline
\end{tabular}

* Distance from the epiphyseal arterial networks (immediately above the scar, not including the arches) to the articular surface of the femoral head; ${ }^{\dagger} \mathrm{p}<0.05$, nonnormal distribution, data for reference only. 


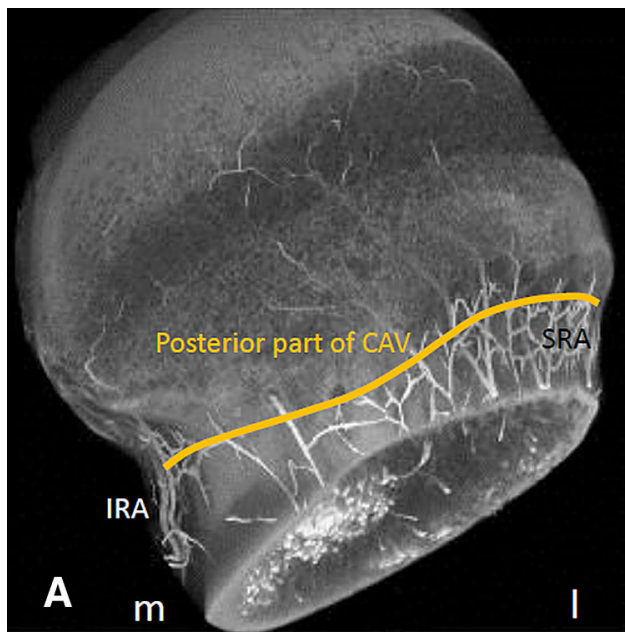

Fig. 6A-B The relationship between the retinacular arterial system and the circulus articuli vasculosus (CAV) around the base of the femoral head is shown. (A) The superior and inferior retinacular arteries joined the posterior part of CAV (orange line) close to the surface of the femoral neck on the cartilaginous edge of the femoral head. (B) The anastomosis among the anterior (dark blue line) and

intraosseous vascular system [4, 20-23]. The most representative research is the study by Trueta and Harrison [21], which showed that different sets of arteries supplied blood to different parts of the femoral head. Some studies provided fragmentary evidence of anastomosis among different regions of the intraosseous vascular system based on the Spalteholz technique [4, 20-23]. Additionally, in a cadaver study, Boraiah et al. [2] confirmed the intraosseous anastomosis between the medial femoral circumflex arterial system and the round ligament arterial system, and further confirmed the importance of the inferior retinacular artery for femoral head blood perfusion by using gadolinium-enhanced MRI. Nonetheless, to our knowledge no studies have explored detailed 3-D arterial anastomotic networks including all blood supply sources of the femoral head and neck (medial and lateral femoral circumflex arterial system, round ligament arterial system, and intraosseous arterial system). The current study provides the 3-D intraosseous arterial framework of the adult femoral head and related quantified data of the intraosseous vessels for the first time. By combining DSA imaging to determine the features of the intraosseous vascular system, the compensatory blood supply pathway of the femoral head after femoral head fracture can be more clearly delineated.

This study has some limitations. First, we did not perform any intra- or interobserver reliability testing in either the cadaver study or the evaluation of the DSA imaging; this may influence the objectivity of some observational results, to some extent. However, because the main results, which may affect the conclusions of this study, are intuitive

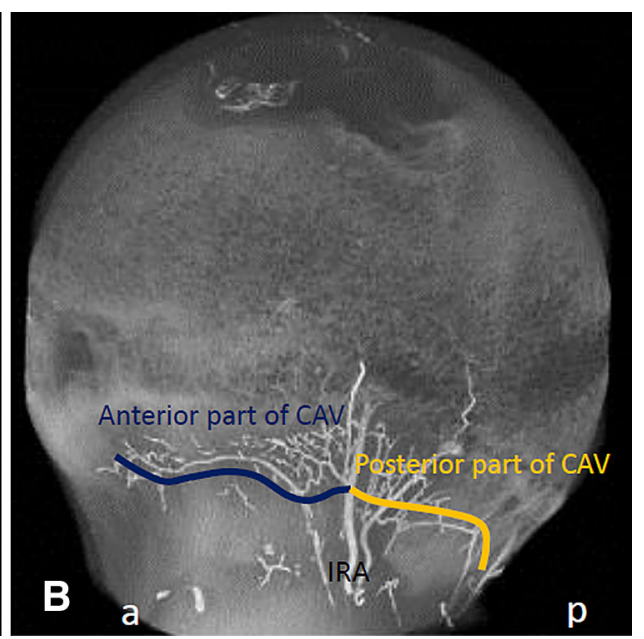

posterior parts (orange line) of the $\mathrm{CAV}$ and inferior retinacular arteries are shown. $\mathrm{m}, \mathrm{l}$, a, and $\mathrm{p}=$ medial, lateral, anterior, and posterior anatomic directions, respectively; SRA = superior retinacular artery; IRA = inferior retinacular artery.

and nonquantitative (such as the position relationship among different intraosseous vascular structures and the DSA imaging of the unaffected retinacular arteries), we believe that these results of consensus have little effect on the final conclusions. Next, the DSA imaging cannot completely reflect the actual damage of the vascular system. Some "affected" vessels still can be revascularized after exclusion of interfering factors (distortions or compression caused by fractures and hematoma). Thus, the actual residual function of the blood vessel after femoral neck fracture is more optimistic than that presented by DSA. In addition, there is no postoperative or followup DSA imaging in this study, and therefore, the extraosseous characteristics of the vascular supply and related potential for revascularization could not be considered. This means that the blood supply situation of femoral head after femoral neck fracture is dynamic. The factors which determine whether secondary necrosis occurs are more complicated than those mentioned in our study. In addition, our study could not provide evidence that the residual blood flow of the inferior retinacular arterial system provides adequate perfusion for the femoral head independently. In the animal model study by Nishino et al. [17], they suggested that a decrease in femoral head blood flow to less than $20 \%$ of the control value is needed to cause osteonecrosis. In addition, Boraiah et al. [2] reported that the inferior retinacular arterial system could provide perfusion independently of the entire femoral head (greater than 20\%). However, there is still a lack of long-term clinical studies regarding this important point. In addition, the fresh cadaver specimens used in the current anatomic 

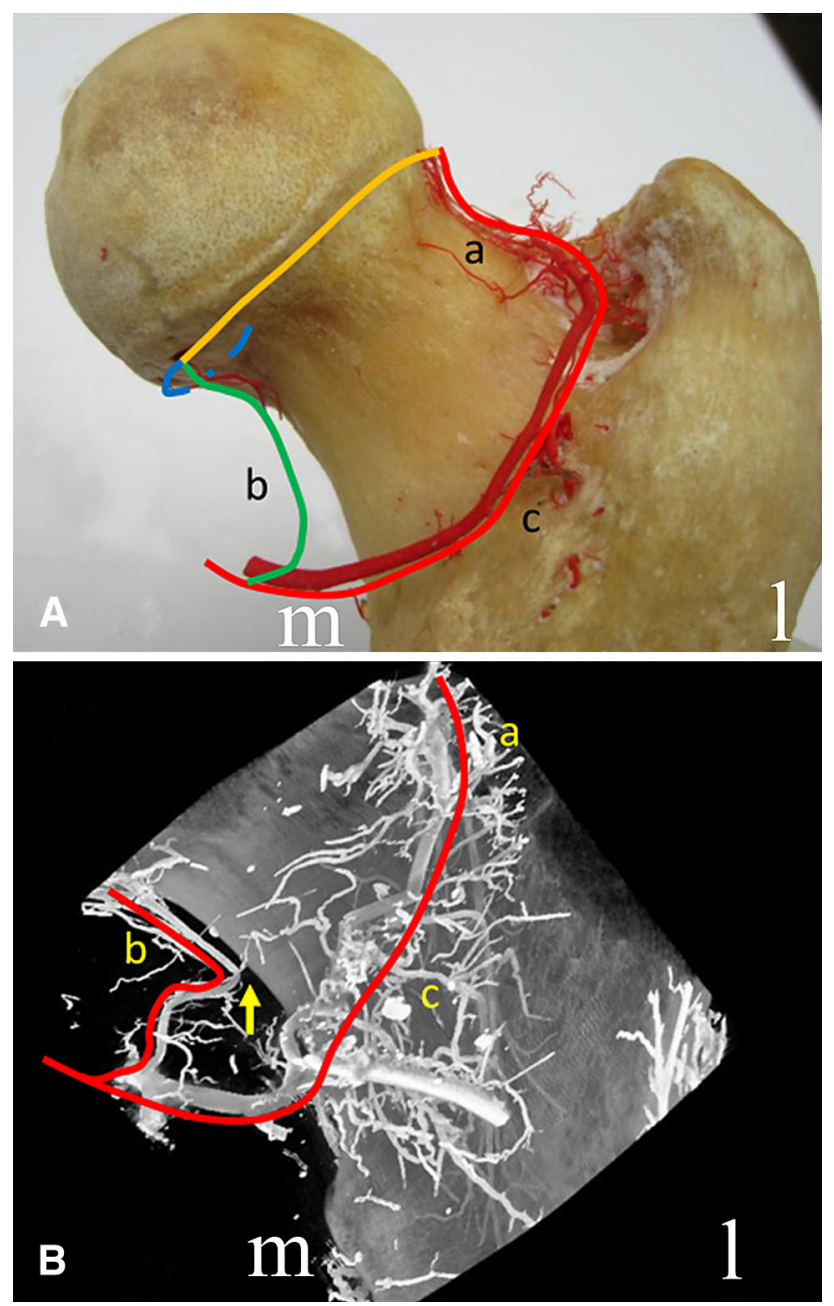

Fig. 7A-B The morphologic characteristics of the "C-shaped ring" and the circulus articuli vasculosus (CAV) around the femoral neck using (A) corrosion casts and (B) angiography are shown (solid red and green lines, A; solid red line. B). The dashed blue and solid yellow lines in Illustration A indicate the anterior and posterior portions of the $\mathrm{CAV}$, respectively; $\mathrm{a}-\mathrm{c}=$ superior and inferior retinacular arteries and the deep branch of the medial femoral circumflex arteries respectively. The yellow arrow in Illustration B points to the gap between the inferior retinacular artery (IRA) and the femoral neck. $\mathrm{m}=$ medial anatomic direction; $1=$ lateral anatomic direction.

study were all of Chinese ethnicity. We consider that the general morphologic characteristics of vascular structures are likely to be universal among different races except for some size-related differences, such as the mean diameter of the superior and inferior retinacular arteries $(0.66 \mathrm{~mm}$ and $0.62 \mathrm{~mm}$, respectively in our study, versus $0.84 \mathrm{~mm}$ and $0.41 \mathrm{~mm}$, respectively in Tucker's study [22]); however, it cannot be ruled out that these differences are caused by racial differences. Finally, because of the shrinkage of the gelatin during hardening, the measurement results of intraand extraosseous vessels may be smaller than the actual values.
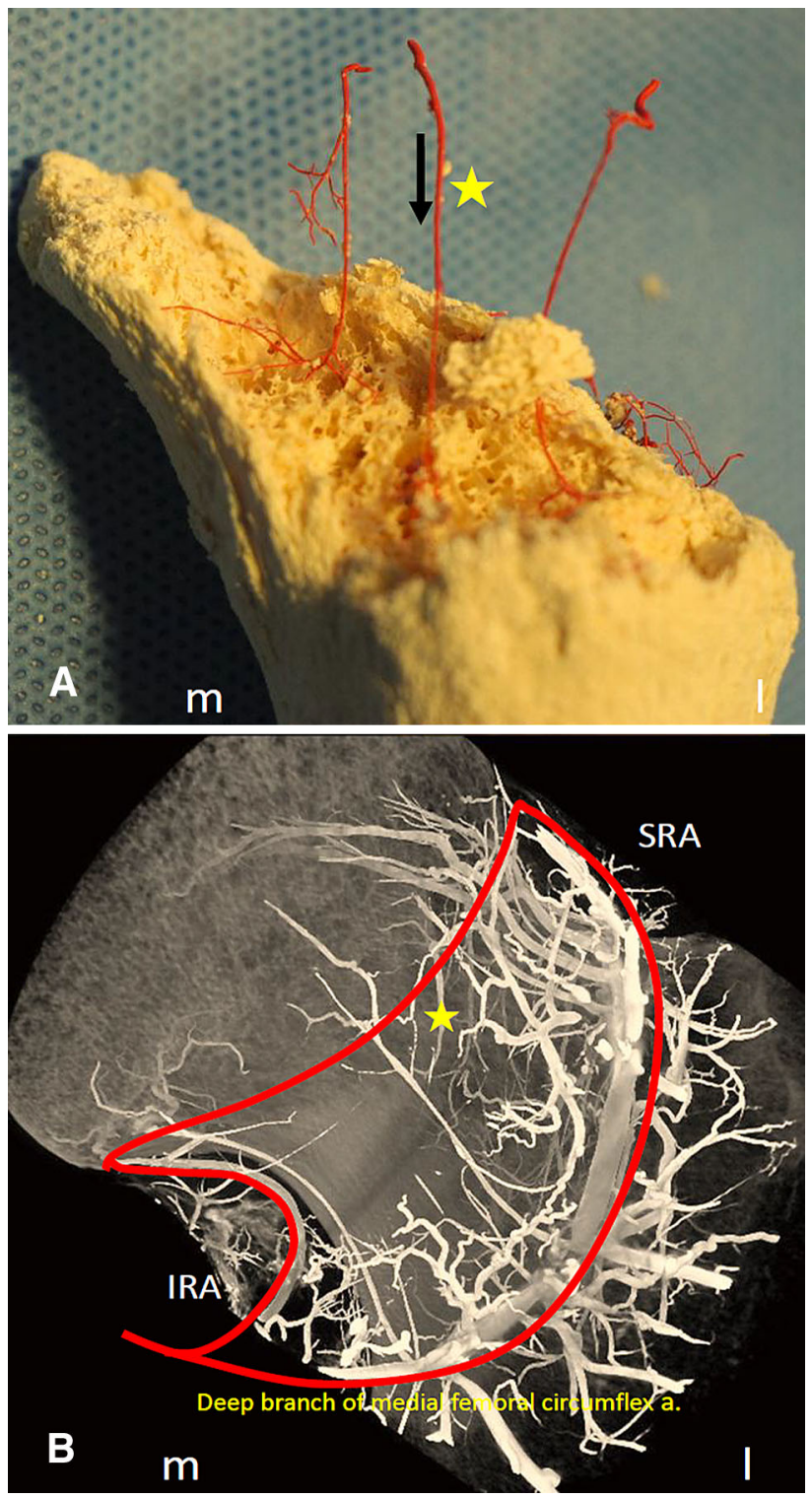

Fig. 8A-B The "C-shaped ring" and the circulus articuli vasculosus (CAV, traced by the red line) radiate as vertically straight vessels (stars) inside the femoral neck bone as shown in the (A) corrosion cast and by (B) angiography. The arrow in Illustration $\mathrm{A}$ indicates the direction of blood flow. $\mathrm{m}=$ medial anatomic direction; $1=$ lateral anatomic direction; SRA = superior retinacular artery; IRA = inferior retinacular artery.

Novel observations in the current study include the following: (1) the main blood supply of the femoral head (superior, inferior, anterior retinacular arteries, round ligament arteries, and intraosseous nutrient arteries of the femur, which correspond with previous studies $[7,12,22,24])$ is connected by three main network structures as a whole: the epiphyseal arterial network and the metaphyseal arterial network in the femoral head and the circulus articuli vasculosus on the neck of the femur. The epiphyseal arterial network structure, located above 


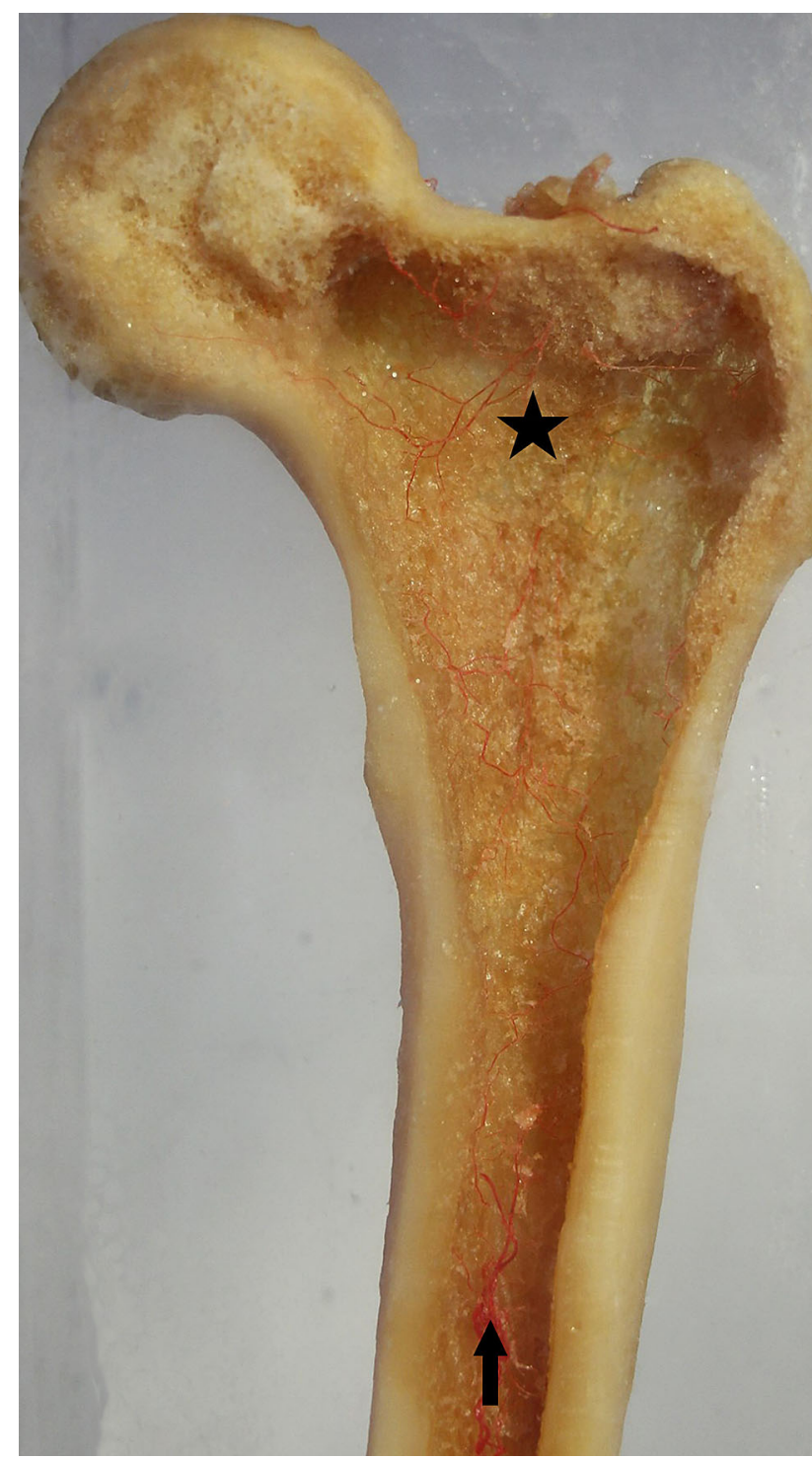

Fig. 9 The femoral nutrient (arrow) and neck (star) arteries anastomose to facilitate blood flow to the femoral head in this corrosion cast showing the proximal intraosseous blood vessels.

the epiphyseal scar, and directly connecting the three groups of the retinacular arterial system and the round ligament arterial system, is the most widely distributed and the primary network structure in the femoral head; (2) generally, structures that are closer to the center of the epiphyseal arterial network exhibit more anastomoses; however, the superolateral region has fewer secondary anastomoses and arches more than the other regions of the femoral head; (3) similar to the superior retinacular artery, the inferior retinacular artery also has a large caliber, whereas the round ligament artery and anterior retinacular artery have a smaller diameter and increased rate of absence; (4) the inferior retinacular arterial system had a substantially increased chance of being unaffected after Garden Types I to III femoral neck fractures (Table 2).
The results of this study suggest that the epiphyseal arterial network and the inferior retinacular arterial systems could be two important structures for maintaining femoral head blood supply after femoral neck fracture. The "high residual rate" characteristic of the inferior retinacular artery suggests that femoral head blood supply after injury is likely maintained through the inferior retinacular arteries-inferior epiphyseal arteries-epiphyseal arterial network and inferior retinacular arteries-metaphyseal arterial network-epiphyseal arterial network pathways and plays an important compensatory role after femoral neck fracture. Several studies have shown that the vessels of the superior retinacular arterial system, as the primary blood supply to the femoral head, constitute the largest and most important retinacular system $[10,11,22]$. Our study indicated that the inferior retinacular artery also had a relatively large caliber (mean diameter, $0.62 \mathrm{~mm}$ ), indicating that the inferior retinacular arterial system could play a more-important role in the blood supply than previously thought [22].

This information suggests that a femoral-head preservation strategy could be applied to a large portion of patients (especially younger patients) with Garden Types I to III femoral neck fractures to avoid further damage to the intra- and extraosseous vascular systems. However, it is notable that the femoral head intraosseous vascular system (especially the epiphyseal arterial network) would inevitably be damaged to various degrees during the process of cannulated screw placement. In a prospective, randomized trial, Lykke et al. [14] compared the use of three Ullevaal hip screws (Orthovita, Oslo, Norway) with that of two Hansson hook-pins (Swemac, Linköping, Sweden) in 278 patients with femoral neck fractures. The evidence indicated a correlation between the quantity of drilling and screwing and the incidence rate of secondary osteonecrosis. Razik et al. [18] reported that the cannulated screw fixation group exhibited an increased risk of osteonecrosis compared with the dynamic hip screw group, suggesting that the method of internal fixation may be related to osteonecrosis. Would a different quantity or location of internal fixation affect the intraosseous vascular system at different levels during the drilling and screw placement procedures? From a morphologic perspective, as shown in our images and video, the main stems of the epiphyseal arteries were located on the periphery of the intraosseous vascular system. The stems have a larger caliber and fewer anastomoses than the network located in the central region. Additionally, the superolateral region (the weightbearing region) has fewer secondary anastomoses. Thus, this region is more vulnerable to the effect of iatrogenic damage during cannulated screw-placement surgery. Based on these characteristics, we suggest that the central region of the femoral head should be a relatively safer area than the peripheral region during drilling and screw placement (to 
Table 2. Assessment on residual blood supply after femoral neck fracture in adults based on the preoperative digital subtraction angiography

\begin{tabular}{|c|c|c|c|c|c|c|}
\hline \multirow[t]{2}{*}{ Patient } & \multirow[t]{2}{*}{ Gender } & \multirow[t]{2}{*}{ Age (years) } & \multirow[t]{2}{*}{ Garden Types I-IV } & \multicolumn{3}{|l|}{ Residual retinacular artery } \\
\hline & & & & Superior retinacular artery & Inferior retinacular artery & Anterior retinacular artery \\
\hline 1 & $\mathrm{~F}$ & 50 & I & Unaffected & Unaffected & Affected \\
\hline 2 & M & 55 & I & Unaffected & Unaffected & Unaffected \\
\hline 3 & $\mathrm{~F}$ & 52 & I & Unaffected & Unaffected & Unaffected \\
\hline 4 & $\mathrm{~F}$ & 32 & I & Unaffected & Unaffected & Unaffected \\
\hline 5 & M & 54 & II & Affected & Unaffected & Affected \\
\hline 6 & M & 38 & II & Affected & Unaffected & Unaffected \\
\hline 7 & $\mathrm{~F}$ & 54 & II & Affected & Unaffected & Affected \\
\hline 8 & $\mathrm{~F}$ & 60 & II & Unaffected & Unaffected & Unaffected \\
\hline 9 & $\mathrm{~F}$ & 52 & II & Unaffected & Unaffected & Unaffected \\
\hline 10 & F & 19 & II & Unaffected & Unaffected & Unaffected \\
\hline 11 & $\mathrm{~F}$ & 60 & II & Unaffected & Unaffected & Unaffected \\
\hline 12 & $\mathrm{~F}$ & 51 & II & Affected & Unaffected & Affected \\
\hline 13 & M & 37 & II & Affected & Unaffected & Affected \\
\hline 14 & M & 71 & II & Unaffected & Unaffected & Unaffected \\
\hline 15 & M & 40 & III & Affected & Affected & Affected \\
\hline 16 & M & 35 & III & Affected & Unaffected & Unaffected \\
\hline 17 & M & 25 & III & Affected & Affected & Affected \\
\hline 18 & $\mathrm{~F}$ & 53 & III & Affected & Unaffected & Affected \\
\hline 19 & M & 52 & III & Affected & Unaffected & Affected \\
\hline 20 & $\mathrm{~F}$ & 41 & III & Affected & Unaffected & Affected \\
\hline 21 & M & 44 & III & Affected & Affected & Affected \\
\hline 22 & $\mathrm{~F}$ & 67 & III & Affected & Unaffected & Unaffected \\
\hline 23 & M & 60 & III & Affected & Affected & Affected \\
\hline 24 & $\mathrm{~F}$ & 25 & III & Affected & Unaffected & Unaffected \\
\hline 25 & M & 51 & IV & Affected & Affected & Affected \\
\hline 26 & M & 50 & IV & Affected & Affected & Affected \\
\hline 27 & $\mathrm{~F}$ & 76 & IV & Affected & Affected & Affected \\
\hline
\end{tabular}

avoid damage to the main stems of epiphyseal arteries and the lateral region of the epiphyseal arterial network) (Fig. 10). Under the premise of a similar Garden type (especially when one or more groups of retinacular arterial systems were affected), with good reduction and fixation stability, do the methods of central internal fixation (such as dynamic hip screws) provide a lower risk of vascular damage than methods that use peripheral internal fixation (such as the use of three screws that form an inverted triangle when placed in the femoral head parallel to the femoral neck long axis) and result in a different prognosis? This seems a subject that deserves more clinical studies. Additionally, the reconstruction and simulation technique used in our study can only observe and detail the intraosseous vascular injury caused by internal fixation in different regions (Fig. 10), however, the extent of this type of destruction (the drilling depth, orientation, and quantity) might be directly related to secondary osteonecrosis, which remains an interesting subject that should be further explored and studied.

We delineated the 3-D morphologic features among the retinacular arteries, round ligament arteries, and intraosseous arterial network in the femoral epiphysis, metaphysis, and neck. Our study showed that the inferior retinacular arterial system has a relatively large caliber and is less affected after femoral neck fractures; thus, the inferior retinacular arterial system and epiphyseal arterial network could be two important structures for maintaining the femoral head blood supply after femoral neck fractures. Increased efforts to protect these key structures of the retinacular and intraosseous blood supply systems during surgery (such as drilling and placing internal implants closer to the central region of the femoral head) might be helpful to reduce the effect of iatrogenic injury of the intraosseous vascular system, but future studies will need to evaluate the clinical relevance of this suggestion. 


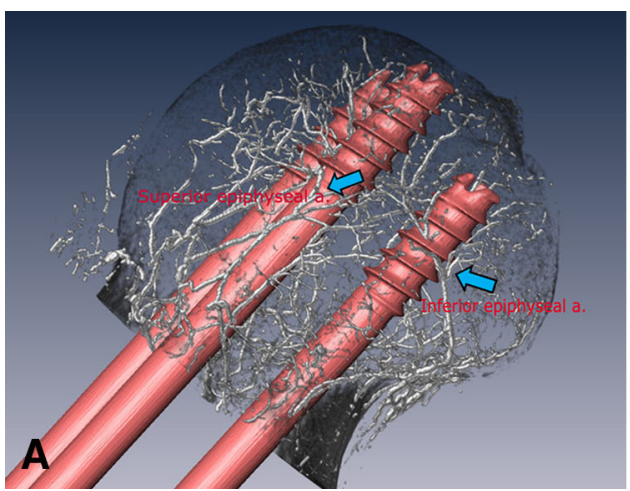

Fig. 10A-B Three-dimensional screw-setting simulations of the intraosseous vascular damage of central and peripheral internal fixation are shown. (A) With peripheral internal fixation, there is a high opportunity to damage the main stems of the epiphyseal arteries, which have a larger caliber and fewer anastomoses than the network located in the central region. (B) In contrast, central internal fixation

Acknowledgments We thank Suzette Smiley-Jewell PhD (Center for Health and the Environment, University of California, Davis, CA, USA) and Simiao Tian PhD (Department of Scientific Research Project, Affiliated Zhongshan Hospital of Dalian University, Liaoning, Dalian, China) for their generous help with technical support.

\section{References}

1. Arnoldi CC, Lemperg RK. Fracture of the femoral neck: II. Relative importance of primary vascular damage and surgical procedure for the development of necrosis of the femoral head. Clin Orthop Relat Res. 1977;129:217-222.

2. Boraiah S, Dyke JP, Hettrich C, Parker RJ, Miller A, Helfet D, Lorich D. Assessment of vascularity of the femoral head using gadolinium (Gd-DTPA)-enhanced magnetic resonance imaging: a cadaver study. J Bone Joint Surg Br. 2009;9:131-137.

3. Catto M. A histological study of avascular necrosis of the femoral head after transcervical fracture. $J$ Bone Joint Surg $\mathrm{Br}$. 1965;47:749-776.

4. Claffey TJ. Avascular necrosis of the femoral head: an anatomical study. J Bone Joint Surg Br 1960;42:802-809.

5. Davidovitch RI, Jordan CJ, Egol KA, Vrahas MS. Challenges in the treatment of femoral neck fractures in the nonelderly adult. $J$ Trauma. 2010;68:236-242.

6. Ehlinger M, Moser T, Adam P, Bierry G, Gangi A, de Mathelin M, Bonnomet F. Early prediction of femoral head avascular necrosis following neck fracture. Orthop Traumatol Surg Res. 2011;97:79-88.

7. Gautier E,Ganz K,Krugel N,Gill T, Ganz R.Anatomy of the medial femoral circumflex artery and its surgical implications. $J$ Bone Joint Surg Br. 2000;82:679-683.

8. Hunter W. Of the structure and diseases of articulating cartilages. Phil Trans. 1742;42:514-521. doi:10.1098/rstl.1742.0079.

9. Judet J, Judet R, Lagrange J, Dunoyer J. A study of the arterial vascularization of the femoral neck in the adult. $J$ Bone Joint Surg Am. 1955;37:663-680.

10. Kalhor M, Beck M, Huff TW, Ganz R. Capsular and pericapsular contributions to acetabular and femoral head perfusion. $J$ Bone Joint Surg Am. 2009;91:409-418.

11. Kalhor M, Horowitz K, Gharehdaghi J, Beck M, Ganz R. Anatomic variations in femoral head circulation. Hip Int. 2012;22:307-312.

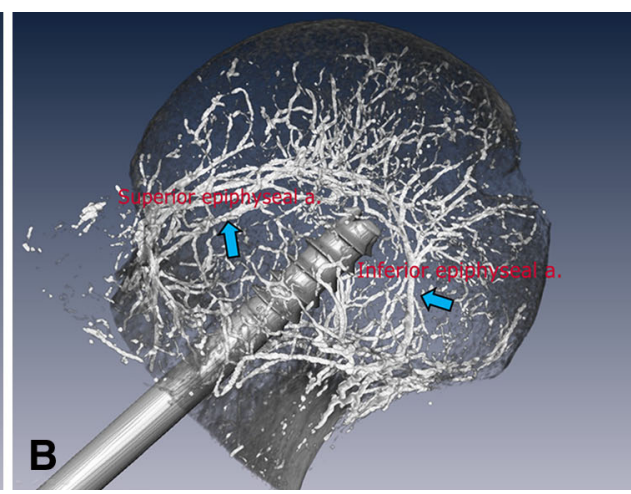

could avoid damaging these main stems of the epiphyseal arteries, and directly face the central region of the epiphyseal arterial network, which has more anastomoses and accordingly higher compensatory capacity. The blue arrows indicate the main stems of superior and inferior epiphyseal arteries.

12. Lazaro LE, Klinger CE, Sculco PK, Helfet DL, Lorich DG. The terminal branches of the medial femoral circumflex artery: the arterial supply of the femoral head. Bone Joint J. 2015;97:12041213

13. Ly TV, Swiontkowski MF. Treatment of femoral neck fractures in young adults. J Bone Joint Surg Am. 2008;90:2254-2266.

14. Lykke N, Lerud PJ, Strømsøe K, Thorngren KG. Fixation of fractures of the femoral neck: a prospective, randomised trial of three Ullevaal hip screws versus two Hansson hook-pins. $J$ Bone Joint Surg Br. 2003;85:426-430.

15. Miyamoto RG, Kaplan KM, Levine BR, Egol KA, Zuckerman JD. Surgical management of hip fractures: an evidence-based review of the literature. I: femoral neck fractures. J Am Acad Orthop Surg. 2008;16:596-607.

16. Nikolopoulos KE, Papadakis SA, Kateros KT, Themistocleous GS, Vlamis JA, Papagelopoulos PJ, Nikiforidis PA. Long-term outcome of patients with avascular necrosis, after internal fixation of femoral neck fractures. Injury. 2003;34:525-528.

17. Nishino M, Matsumoto T, Nakamura T, Tomita K. Pathological and hemodynamic study in a new model of femoral head necrosis following traumatic dislocation. Arch Orthop Trauma Surg. 1997; 116:259-262.

18. Razik F, Alexopoulos AS, El-Osta B, Connolly M, Brown A, Hassan S, Ravikumar K. Time to internal fixation of femoral neck fractures in patients under sixty years: does this matter in the development of osteonecrosis of femoral head? Int Orthop. 2012;36:2127-2132

19. Reinhold HS, Hopewell JW, Van Rijsoort A. A revision of the Spalteholz method for visualizing blood vessels. Int J Microcirc Clin Exp. 1983;2:47-52.

20. Sevitt S, Thompson RG. The distribution and anastomoses of arteries supplying the head and neck of the femur. J Bone Joint Surg Br. 1965;47:560-573.

21. Trueta J, Harrison MH. The normal vascular anatomy of the femoral head in adult man. J Bone Joint Surg Br. 1953;35:442461.

22. Tucker FR. Arterial supply to the femoral head and its clinical importance. J Bone Joint Surg Br. 1949;31:82-93.

23. Wertheimer LG, Lopes Sde L. Arterial supply of the femoral head: a combined angiographic and histological study. J Bone Joint Surg Am. 1971;53:545-556.

24. Zlotorowicz M, Szczodry M,Czubak J,Ciszek B.Anatomy of the medial femoral circumflex artery with respect to the vascularity of the femoral head. J Bone Joint Surg Br. 2011;93:1471-1474. 